scoliosis and on 38\% of idiopathic scoliosis. E.E.G. was in borderline on $28 \%$ of idiopathic scoliosis. E.E.G. was abnormal on $55 \%$ and more of the prescoliotic stage and on $30 \%$ of prescoliosis.

Some cases of so-called idiopathic scoliosis showed neu rological symptoms including abducense nerve palsy, nystagmus, and strabismus. These findings suggest that some cases of idiopathic scoliosis come from a disorder in the central nervous system and some cases of paralytic scoliosis come from not only poliomyelitis but also disorders within the brain.

\title{
98. Experimental Studies on the Fiber Connections Related to the Nystagmus
}

\author{
Tadayasu Ban, Shozo Matano \\ and Katsuya Zyo \\ Department of Anatomy, Osaka University Medical School
}

Marchi's preparations of 60 rabbit brains were used for these studies.

1) On the optokinetic mystagmus: Descending tectofugal fibers terminated in the interstitial nuclei of Cajal, oculomotor nuclei and the trochlear nuclei on both sides. The tectospinal tract formed on the contralateral side, ramified in the abducens nucleus on its side and was followed to the cervical cord.

2) On the vestibular nystagmus: Fibers from the superior, medial, lateral and the inferior vestibular nuclei terminated in the trochlear nuclei, oculomotor nuclei and the interstitial nuclei of Cajal on both sides via the medial longitudinal fasciculus. Fibers from the medial, lateral and the inferior vestibular nuclei terminated in the abducens nuclei on both sides, but fibers from the superior vestibular nucleus terminated in the abducens nucleus on the same side.

3) On the cerebellar nystagmus: Cerebellofugal fibers via the superior cerebellar peduncle terminated in the trochlear nuclei, oculomotor nuclei and the interstitial nuclei of Cajal on both sides. In a human brain, of which the fastigial nuclei and the vermal white matter, were destructed, degenerated fibers entered the superior, medial and the lateral vestibular nuclei and the abducens nucleus, and moreover, other degenerated fibers terminated in the trochlear, oculomotor and the interstitial nuclei of Cajal via the FLM.

4) On the mesencephalic component of FLM: Descending fibers in 
both FLMs originated chiefly in the interstitial nuclei of Cajal entered the oculomotor and trochlear nuclei on both sides and the abducens nucleus on the same side. And the posterior commissure connects the interstitial nuclei of Cajal of each side.

The disturbance of balance in these fiber systems mentioned above is considered to have a close relationship with occurrence of the nystagmus. And the interstitial nucleus of Cajal, which is an important intermediate station of these systems, receives fibers from the autonomic nervous system and the nucleus originates the extrapyramidal fibers. Furthermore, many fine fibers from the vestibular nuclei, cerebellar nuclei and the superior colliculus proceed into the dorsal longitudinal fasciculus. These fiber connections might become a background of the nystagmus.

\title{
99. Studies on the Inhibitory and Facilitatory Mechanisms of Neurons in the Nucleus Tractus Solitarius of Cat
}

\author{
Miyoshi Urabe, Takashi Tsubokawa, Yoh Watanabe, \\ Noboru Hamabe, Haruhide Ito \\ and Shuji Asano \\ Department of Surgery, School of Medicine, University of Kanazawa
}

Our previous investigation suggested that higher levels of central nervous system might exert inhibitory influences on the viscerosensory impulses of the vagal origin ascending to the thalamic nuclei through the nucleus tractus solitarius.

To cralify this problem, experiments were performed on 19 nonanesthetized cats. Extracelluar recording from the nucleus tractus solitarius (NTS) carried out stereotaxically using tungsten microelectrodes. Single and repititive electrical shockes were applied to the central cut end of the cervical vagus nerve, pericuruciate area of the cerbral cortex and midbrain reticular formation (MRF). The analysis of the observations were done on 67 units which were identified as the neurons belonging to the NTS.

The results are as follows.

1) Latencies of the initial spike of the units which could be activated by vagal stimulation, ranged widely from $2 \mathrm{msec}$ to $20 \mathrm{msec}$. The units having been affected from the cortex and MRF had the latencies of longer than $10 \mathrm{msec}$.

2) On 41 units, the effect of repititive stimulation of the cortex and 